This postprint is published in:

Mathematica slovaca, Volume 65, Number 5, 2015, 981-992

DOI: http://dx.doi.org/10.1515/ms-2015-0067

\title{
ON MONOIDS OF INJECTIVE PARTIAL COFINITE SELFMAPS
}

\author{
OLEG GUTIK AND DUŠAN REPOVŠ
}

\begin{abstract}
We study the semigroup $\mathcal{J}_{\lambda}^{c f}$ of injective partial cofinite selfmaps of an infinite cardinal $\lambda$. We show that $\mathcal{J}_{\lambda}^{\mathrm{cf}}$ is a bisimple inverse semigroup and each chain of idempotents in $\mathcal{J}_{\lambda}^{\mathrm{ff}}$ is contained in a bicyclic subsemigroup of $\mathcal{J}_{\lambda}^{\text {cf }}$, we describe the Green relations on $\mathcal{J}_{\lambda}^{\text {cf }}$ and we prove that every non-trivial congruence on $\mathcal{J}_{\lambda}^{\text {cf }}$ is a group congruence. Also, we describe the structure of the quotient semigroup $\mathcal{J}_{\lambda}^{c f} / \sigma$, where $\sigma$ is the least group congruence on $\mathcal{J}_{\lambda}^{\text {cf }}$.
\end{abstract}

\section{INTRODUCTION AND PRELIMINARIES}

In this paper we shall denote the first infinite ordinal by $\omega$ and the cardinality of the set $A$ by $|A|$. We shall identify all cardinals with their corresponding initial ordinals. We shall denote the set of integers by $\mathbb{Z}$ and the additive group of integers by $\mathbb{Z}(+)$.

A semigroup $S$ is called inverse if for every element $x \in S$ there exists a unique $x^{-1} \in S$ such that $x x^{-1} x=x$ and $x^{-1} x x^{-1}=x^{-1}$. The element $x^{-1}$ is called the inverse of $x \in S$. If $S$ is an inverse semigroup, then the function inv: $S \rightarrow S$ which assigns to every element $x$ of $S$ its inverse element $x^{-1}$ is called the inversion.

A congruence $\mathfrak{C}$ on a semigroup $S$ is called non-trivial if $\mathfrak{C}$ is distinct from the universal and the identity congruences on $S$, and a group congruence if the quotient semigroup $S / \mathfrak{C}$ is a group.

If $S$ is a semigroup, then we shall denote the subset of all idempotents in $S$ by $E(S)$. If $S$ is an inverse semigroup, then $E(S)$ is closed under multiplication and we shall refer to $E(S)$ as a band (or the band of $S$ ). Then the semigroup operation on $S$ determines the following partial order $\leqslant$ on $E(S): e \leqslant f$ if and only if $e f=f e=e$. This order is called the natural partial order on $E(S)$. A semilattice is a commutative semigroup of idempotents. A semilattice $E$ is called linearly ordered or a chain if its natural order is a linear order. A maximal chain of a semilattice $E$ is a chain which is not properly contained in any other chain of E.

The Axiom of Choice implies the existence of maximal chains in every partially ordered set. According to [13, Definition II.5.12], a chain $L$ is called an $\omega$-chain if $L$ is isomorphic to $\{0,-1,-2,-3, \ldots\}$ with the usual order $\leqslant$ or equivalently, if $L$ is isomorphic to $(\omega, \max )$. Let $E$ be a semilattice and $e \in E$. We put $\downarrow e=\{f \in$ $E \mid f \leqslant e\}$ and $\uparrow e=\{f \in E \mid e \leqslant f\}$. By $\left(\mathcal{P}_{<\omega}(\lambda), \cup\right)$ we shall denote the free semilattice with identity over a set of cardinality $\lambda \geqslant \omega$, i.e., $\left(\mathcal{P}_{<\omega}(\lambda), \cup\right)$ is the set of all finite subsets (with the empty set) of $\lambda$ with the semilattice operation "union".

2010 Mathematics Subject Classification. Primary 20M20, 20M18; Secondary 20B30.

Key words and phrases. Bicyclic semigroup, semigroup of bijective partial transformations, congruence, symmetric group, group congruence, semidirect product. 
If $S$ is a semigroup, then we shall denote the Green relations on $S$ by $\mathcal{R}, \mathcal{L}, \mathcal{J}$, $\mathcal{D}$ and $\mathcal{H}$ (see [5]). A semigroup $S$ is called simple if $S$ does not contain proper two-sided ideals and bisimple if $S$ has only one $\mathcal{D}$-class.

The bicyclic semigroup $\mathcal{C}(p, q)$ is the semigroup with the identity 1 generated by elements $p$ and $q$ subject only to the condition $p q=1$. The bicyclic semigroup is bisimple and every one of its congruences is either trivial or a group congruence. Moreover, every homomorphism $h$ of the bicyclic semigroup is either an isomorphism or the image of $\mathcal{C}(p, q)$ under $h$ is a cyclic group (see [5. Corollary 1.32]). The bicyclic semigroup plays an important role in algebraic theory of semigroups and in the theory of topological semigroups. For example a well-known Andersen's result [1] states that a (0-) simple semigroup is completely (0-) simple if and only if it does not contain the bicyclic semigroup. The bicyclic semigroup admits only the discrete topology [7. The problem of embeddability of the bicycle semigroup into compact-like semigroups was studied in $2,4,4,4,11$.

Remark 1.1. We observe that the bicyclic semigroup is isomorphic to the semigroup $\mathcal{C}_{\mathbb{N}}(\alpha, \beta)$ which is generated by partial transformations $\alpha$ and $\beta$ of the set of positive integers $\mathbb{N}$, defined as follows: $(n) \alpha=n+1$ if $n \geqslant 1$ and $(n) \beta=n-1$ if $n>1$ (see Exercise IV.1.11(ii) in [13]).

If $T$ is a semigroup, then we say that a subsemigroup $S$ of $T$ is a bicyclic subsemigroup of $T$ if $S$ is isomorphic to the bicyclic semigroup $\mathcal{C}(p, q)$.

Hereafter we shall assume that $\lambda$ is an infinite cardinal. If $\alpha: X \rightarrow Y$ is a partial map, then we shall denote the domain and the range of $\alpha$ by $\operatorname{dom} \alpha$ and $\operatorname{ran} \alpha$, respectively.

Let $\mathcal{J}_{\lambda}$ denote the set of all partial one-to-one transformations of an infinite set $X$ of cardinality $\lambda$ together with the following semigroup operation:

$$
x(\alpha \beta)=(x \alpha) \beta \quad \text { if } \quad x \in \operatorname{dom}(\alpha \beta)=\{y \in \operatorname{dom} \alpha \mid y \alpha \in \operatorname{dom} \beta\}, \quad \text { for } \alpha, \beta \in \mathcal{J}_{\lambda} .
$$

The semigroup $\mathcal{J}_{\lambda}$ is called the symmetric inverse semigroup over the set $X$ (see [5, Section 1.9]). The symmetric inverse semigroup was introduced by Vagner [21] and it plays a major role in the theory of semigroups.

Furthermore, we shall identify the cardinal $\lambda=|X|$ with the set $X$. By $\mathcal{J}_{\lambda}^{\text {cf }}$ we shall denote a subsemigroup of injective partial selfmaps of $\lambda$ with cofinite domains and ranges in $\mathcal{J}_{\lambda}$, i.e.,

$$
\mathcal{J}_{\lambda}^{\text {cf }}=\left\{\alpha \in \mathcal{J}_{\lambda}|| \lambda \backslash \operatorname{dom} \alpha \mid<\infty \text { and }|\lambda \backslash \operatorname{ran} \alpha|<\infty\right\} .
$$

Obviously, $\mathcal{J}_{\lambda}^{c f}$ is an inverse submonoid of the semigroup $\mathcal{J}_{\lambda}$. We shall call the semigroup $\mathcal{J}_{\lambda}^{\mathrm{cf}}$ the monoid of injective partial cofinite selfmaps of $\lambda$.

Next, by $\mathbb{I}$ we shall denote the identity and by $H(\mathbb{I})$ the group of units of the semigroup $\mathcal{J}_{\lambda}^{\mathrm{cf}}$.

It well known that each partial injective cofinite selfmap $f$ of $\lambda$ induces a homeomorphism $f^{*}: \lambda^{*} \rightarrow \lambda^{*}$ of the remainder $\lambda^{*}=\beta \lambda \backslash \lambda$ of the Stone-Čech compactification of the discrete space $\lambda$. Moreover, under some set theoretic axioms (like PFA or OCA), each homeomorphism of $\omega^{*}$ is induced by some partial injective cofinite selfmap of $\omega$ (see [15]-[20]). So the inverse semigroup $\mathcal{J}_{\lambda}^{\text {cf }}$ admits a natural homomorphism $\mathfrak{h}: \mathcal{J}_{\lambda}^{\mathrm{cf}} \rightarrow \mathcal{H}\left(\lambda^{*}\right)$ to the homeomorphism group $\mathcal{H}\left(\lambda^{*}\right)$ of $\lambda^{*}$ and this homomorphism is surjective under certain set theoretic assumptions.

The semigroups $\mathcal{J}_{\infty}^{\nearrow}(\mathbb{N})$ and $\mathcal{J}_{\infty}^{\nearrow}(\mathbb{Z})$ of injective isotone partial selfmaps with cofinite domains and images of positive integers and integers, respectively, were studied 
in [9] and [10. There it was proved that the semigroups $\mathcal{J}_{\infty}^{\nearrow}(\mathbb{N})$ and $\mathcal{J}_{\infty}^{\nearrow}(\mathbb{Z})$ have properties similar to the bicyclic semigroup: they are bisimple and every non-trivial homomorphic image of $\mathcal{J}_{\infty}^{\nearrow}(\mathbb{N})$ and $\mathcal{J}_{\infty}^{\nearrow}(\mathbb{Z})$ is a group, and moreover, the semigroup $\mathcal{J}_{\infty}^{\nearrow}(\mathbb{N})$ has $\mathbb{Z}(+)$ as a maximal group image and $\mathcal{J}_{\infty}^{\nearrow}(\mathbb{Z})$ has $\mathbb{Z}(+) \times \mathbb{Z}(+)$, respectively.

In this paper we shall study algebraic properties of the semigroup $\mathcal{J}_{\lambda}^{\mathrm{cf}}$. We shall show that $\mathcal{J}_{\lambda}^{\text {cf }}$ is a bisimple inverse semigroup and every chain of idempotents in $\mathcal{J}_{\lambda}^{\mathrm{cf}}$ is contained in a bicyclic subsemigroup of $\mathcal{J}_{\lambda}^{\mathrm{cf}}$, we shall describe the Green relations on $\mathcal{J}_{\lambda}^{\mathrm{cf}}$ and we shall prove that every non-trivial congruence on $\mathcal{J}_{\lambda}^{\mathrm{cf}}$ is a group congruence. Also, we shall describe the structure of the quotient semigroup $\mathrm{J}_{\lambda}^{\mathrm{cf}} / \sigma$, where $\sigma$ is the least group congruence on $\mathcal{J}_{\lambda}^{\mathrm{cf}}$.

\section{Algebraic Properties of the Semigroup $\mathcal{J}_{\lambda}^{c f}$}

Proposition 2.1. $\quad$ (i) $\mathrm{J}_{\lambda}^{\mathrm{cf}}$ is a simple semigroup.

(ii) An element $\alpha$ of the semigroup $\mathcal{J}_{\lambda}^{\mathrm{cf}}$ is an idempotent if and only if $(x) \alpha=x$ for every $x \in \operatorname{dom} \alpha$.

(iii) If $\varepsilon, \iota \in E\left(\mathcal{J}_{\lambda}^{\mathrm{cf}}\right)$, then $\varepsilon \leqslant \iota$ if and only if $\operatorname{dom} \varepsilon \subseteq \operatorname{dom} \iota$.

(iv) The semilattice $E\left(\mathcal{J}_{\lambda}^{\mathrm{cf}}\right)$ is isomorphic to $\left(\mathcal{P}_{<\omega}(\lambda), \cup\right)$ under the mapping $(\varepsilon) h=\lambda \backslash \operatorname{dom} \varepsilon$.

(v) Every maximal chain in $E\left(\mathcal{J}_{\lambda}^{\mathrm{cf}}\right)$ is an $\omega$-chain.

(vi) $\alpha \mathcal{R} \beta$ in $\mathcal{J}_{\lambda}^{\mathrm{cf}}$ if and only if $\operatorname{dom} \alpha=\operatorname{dom} \beta$.

(vii) $\alpha \mathcal{L} \beta$ in $\mathcal{J}_{\lambda}^{\mathrm{cf}}$ if and only if $\operatorname{ran} \alpha=\operatorname{ran} \beta$.

(viii) $\alpha \mathcal{H} \beta$ in $\mathcal{J}_{\lambda}^{\text {cf }}$ if and only if $\operatorname{dom} \alpha=\operatorname{dom} \beta$ and $\operatorname{ran} \alpha=\operatorname{ran} \beta$.

(ix) $\alpha \mathcal{D} \beta$ for all $\alpha, \beta \in \mathcal{J}_{\lambda}^{\mathrm{cf}}$ and hence the semigroup $\mathcal{J}_{\lambda}^{\mathrm{cf}}$ is bisimple.

Proof. (i) We shall show that $\mathcal{J}_{\lambda}^{\mathrm{cf}} \cdot \alpha \cdot \mathcal{J}_{\lambda}^{\mathrm{cf}}=\mathcal{J}_{\lambda}^{\mathrm{cf}}$ for every element $\alpha \in \mathcal{J}_{\lambda}^{\mathrm{cf}}$. Let $\alpha$ and $\beta$ are arbitrary elements of the semigroup $\mathcal{J}_{\lambda}^{\text {cf }}$. We shall choose elements $\gamma, \delta \in \mathcal{J}_{\lambda}^{\text {cf }}$ such that $\gamma \cdot \alpha \cdot \delta=\beta$. We put $\operatorname{dom} \gamma=\operatorname{dom} \beta, \operatorname{ran} \gamma=\operatorname{dom} \alpha, \operatorname{dom} \delta=\operatorname{ran} \alpha$ and $\operatorname{ran} \delta=\operatorname{ran} \beta$. Since the sets $\lambda \backslash \operatorname{dom} \alpha$ and $\lambda \backslash \operatorname{dom} \beta$ are finite we conclude that there exists a bijective map $f: \operatorname{dom} \alpha \rightarrow \operatorname{dom} \beta$. We put $\gamma=f$ and $(((x) \gamma) \alpha) \delta=(x) \beta$ for all $x \in \operatorname{dom} \beta$. Then we have that $\gamma \cdot \alpha \cdot \delta=\beta$.

Statements $(i i)-(v)$ are trivial and they follow from the definition of the semigroup $\mathcal{J}_{\lambda}^{\mathrm{cf}}$. The proofs of $(v i)-(v i i i)$ follow trivially from the fact that $\mathcal{J}_{\lambda}^{\mathrm{cf}}$ is a regular semigroup, and Proposition 2.4.2 and Exercise 5.11.2 in [12].

(ix) Let $\alpha$ and $\beta$ be arbitrary elements of the semigroup $\mathcal{J}_{\lambda}^{\mathrm{cf}}$. Since the sets $\lambda \backslash \operatorname{dom} \alpha$ and $\lambda \backslash \operatorname{ran} \beta$ are finite we conclude that there exists a bijective map $\gamma: \operatorname{dom} \alpha \rightarrow \operatorname{ran} \beta$. Then $\gamma \in \mathcal{J}_{\lambda}^{\text {cf }}$ and by statements (vi) and (vii) we have that $\alpha \mathcal{R} \gamma$ and $\beta \mathcal{L} \gamma$ in $\mathcal{J}_{\lambda}^{\text {cf }}$ and hence $\alpha \mathcal{D} \beta$ in $\mathcal{J}_{\lambda}^{\text {cf }}$.

We denote the group of all bijective transformations of a set of cardinality $\lambda$ by $\mathcal{S}_{\lambda}$. Then we get the following:

Corollary 2.2. The group of units $H(\mathbb{I})$ of the semigroup $\mathcal{J}_{\lambda}^{\mathrm{cf}}$ is isomorphic to $\mathcal{S}_{\lambda}$.

For any idempotents $\varepsilon$ and $\iota$ of the semigroup $\mathcal{J}_{\lambda}^{\text {cf }}$ we denote:

$$
H(\varepsilon, \iota)=\left\{\chi \in \mathcal{J}_{\lambda}^{\mathrm{cf}} \mid \chi \cdot \chi^{-1}=\varepsilon \text { and } \chi^{-1} \cdot \chi=\iota\right\} \quad \text { and } \quad H(\varepsilon)=H(\varepsilon, \varepsilon) .
$$

Proposition 2.1(viii) implies that the set $H(\varepsilon, \iota)$ is a $\mathcal{H}$-class and the set $H(\varepsilon)$ is a a maximal subgroup in $\mathcal{J}_{\lambda}^{\text {cf }}$ for all idempotents $\varepsilon, \iota \in \mathcal{J}_{\lambda}^{\text {cf }}$.

Corollary 2.2 and Proposition 2.20 of [5] imply the following: 
Corollary 2.3. Every maximal subgroup of the semigroup $\mathcal{J}_{\lambda}^{\mathrm{cf}}$ is isomorphic to $\mathcal{S}_{\lambda}$.

Proposition 2.4. $\left|\mathcal{J}_{\lambda}^{\mathrm{cf}}\right|=2^{|\lambda|}$.

Proof. Since $|\lambda \times \lambda|=|\lambda|$ we have that $\left|\mathcal{S}_{\lambda}\right| \leqslant 2^{|\lambda \times \lambda|}=2^{|\lambda|}$. Since $|\lambda \sqcup \lambda|=|\lambda|$ there exists an injective map $f: \mathcal{P}(\lambda) \rightarrow \mathcal{S}_{\lambda \sqcup \lambda}$ from the set $\mathcal{P}(\lambda)$ of all subset of the cardinal $\lambda$ into the group $\mathcal{S}_{\lambda \sqcup \lambda}$ defined in the following way: $f(A)$ is a bijection on $\lambda \sqcup \lambda$ with support $A \sqcup A$. Then we have that $\left|\mathcal{S}_{\lambda}\right| \geqslant 2^{|\lambda \sqcup \lambda|}=2^{|\lambda|}$ and hence $\left|\mathcal{S}_{\lambda}\right|=2^{|\lambda|}$.

Since $\left|\mathcal{P}_{<\omega}(\lambda)\right|=\left|\mathcal{P}_{<\omega}(\lambda) \times \mathcal{P}_{<\omega}(\lambda)\right|=\lambda$ we conclude that Theorem 2.20 from [5] and Proposition 2.1 (viii) imply that

$$
\left|\mathcal{J}_{\lambda}^{\text {cf }}\right|=\left|\mathcal{P}_{<\omega}(\lambda) \times \mathcal{P}_{<\omega}(\lambda) \times \mathcal{S}_{\lambda}\right|=\left|\mathcal{P}_{<\omega}(\lambda) \times \mathcal{P}_{<\omega}(\lambda)\right| \cdot\left|\mathcal{S}_{\lambda}\right|=|\lambda| \cdot 2^{|\lambda|}=2^{|\lambda|} .
$$

Proposition 2.5. For every $\alpha, \beta \in \mathcal{J}_{\lambda}^{\mathrm{cf}}$, both sets

$$
\left\{\chi \in \mathcal{J}_{\lambda}^{\mathrm{cf}} \mid \alpha \cdot \chi=\beta\right\} \quad \text { and } \quad\left\{\chi \in \mathcal{J}_{\lambda}^{\mathrm{cf}} \mid \chi \cdot \alpha=\beta\right\}
$$

are finite. Consequently, every right translation and every left translation by an element of the semigroup $\mathcal{J}_{\lambda}^{\mathrm{cf}}$ is a finite-to-one map.

Proof. We denote

$$
A=\left\{\chi \in \mathcal{J}_{\lambda}^{\mathrm{cf}} \mid \alpha \cdot \chi=\beta\right\} \quad \text { and } B=\left\{\chi \in \mathcal{J}_{\lambda}^{\mathrm{cf}} \mid \alpha^{-1} \cdot \alpha \cdot \chi=\alpha^{-1} \cdot \beta\right\} .
$$

Then $A \subseteq B$ and the restriction of any partial map $\chi \in B$ onto $\operatorname{dom}\left(\alpha^{-1} \cdot \alpha\right)$ coincides with the partial map $\alpha^{-1} \cdot \beta$. Since every partial map from $\mathcal{J}_{\lambda}^{\text {cf }}$ has cofinite range and cofinite domain we conclude that the set $B$ is finite and hence so is $A$.

Proposition 2.6. Each maximal chain $L$ of idempotents in $\mathcal{J}_{\lambda}^{\mathrm{cf}}$ coincides with the idempotent band $E(S)$ of a bicyclic subsemigroup $S$ of $\mathcal{J}_{\lambda}^{c f}$.

Proof. By Proposition 2.1 (iii), the chain $L$ can be written as $L=\left\{\varepsilon_{n}\right\}_{n=1}^{\infty}$ where $\varepsilon_{1}>\varepsilon_{2}>\cdots>\varepsilon_{n}>\cdots$. Since every infinite subchain of an $\omega$-chain is also an $\omega$-chain we have that Proposition 2.1 (v) implies that $L$ is an $\omega$-chain. Then by Proposition 2.1 $($ iii $)$ we get that $\operatorname{dom} \varepsilon_{i} \backslash \operatorname{dom} \varepsilon_{i+1} \neq \varnothing$ for all positive integers $i$. Also, the maximality of $L$ implies that the set $\operatorname{dom} \varepsilon_{i} \backslash \operatorname{dom} \varepsilon_{i+1}$ is a singleton for all positive integers $i$. For every positive integer $i$ we put $\left\{x_{i}\right\}=\operatorname{dom} \varepsilon_{i} \backslash \operatorname{dom} \varepsilon_{i+1}$. Then we put $D=\operatorname{dom} \varepsilon_{1} \backslash \bigcup_{i \in \mathbb{N}}\left\{x_{i}\right\}$ and define the partial maps $\alpha: \lambda \rightarrow \lambda$ and $\beta: \lambda \rightarrow \lambda$ as follows:

$$
(x) \alpha= \begin{cases}x_{n+1}, & \text { if } x=x_{n} \in \operatorname{dom} \varepsilon_{1} \backslash D \text { and } n \geqslant 1 \\ x, & \text { if } x \in D\end{cases}
$$

and

$$
(x) \beta= \begin{cases}x_{n-1}, & \text { if } x=x_{n} \in \operatorname{dom} \varepsilon_{1} \backslash D \text { and } n>1 \\ x, & \text { if } x \in D .\end{cases}
$$

Since the set $\lambda \backslash \operatorname{dom} \varepsilon_{1}$ is finite we have that $\alpha, \beta \in \mathcal{J}_{\lambda}^{\text {cf }}$ and Remark 1.1 implies the statement of our proposition.

Proposition 2.6 and the Axiom of Choice imply the following proposition.

Proposition 2.7. Each chain of idempotents in $\mathcal{J}_{\lambda}^{\mathrm{cf}}$ is contained in a bicyclic subsemigroup of $\mathcal{J}_{\lambda}^{\mathrm{cf}}$. 
Proposition 2.8. Let $\mathfrak{C}$ be a congruence on the semigroup $\mathfrak{J}_{\lambda}^{\mathrm{cf}}$. If there exist two

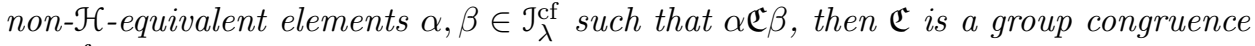
on $\mathcal{J}_{\lambda}^{\mathrm{cf}}$.

Proof. First we suppose that $\alpha$ and $\beta$ are distinct idempotents of the semigroup $\mathcal{J}_{\lambda}^{\text {cf }}$. Without loss of generality we can assume that $\alpha$ and $\beta$ are compatible and $\alpha \leqslant \beta$. Otherwise, replace $\alpha$ by $\alpha \cdot \beta$. Then by Proposition 2.7 there exists a maximal chain $L$ in $E\left(\mathcal{J}_{\lambda}^{\mathrm{cf}}\right)$ such that $L$ contains the elements $\alpha$ and $\beta$, and hence $L$ contained in a bicyclic subsemigroup $S$ of $\mathcal{J}_{\lambda}^{\mathrm{cf}}$. Then Corollary 1.32 of $[5]$ implies that $\varepsilon \mathfrak{C} \iota$ for all elements $\varepsilon$ and $\iota$ of the chain $L$.

Let $\nu$ be an arbitrary idempotent of the semigroup $\mathcal{J}_{\lambda}^{\text {cf }}$. Obviously, if $\varepsilon, \iota \in L$ such that $\varepsilon \leqslant \iota$ then $\varepsilon \cdot \nu \leqslant \iota \cdot \nu$. Since $\uparrow e$ is a finite subset of the free semilattice with unity $\left(\mathcal{P}_{<\omega}(\lambda), \subseteq\right)$ for any $e \in\left(\mathcal{P}_{<\omega}(\lambda), \subseteq\right)$, we have that Proposition 2.1 (iv) implies that $\nu L$ is an infinite chain in $E\left(\mathcal{J}_{\lambda}^{\text {cf }}\right)$. Then we have that $\varepsilon \mathfrak{C} \iota$ for all $\varepsilon, \iota \in \nu L$. We put $L_{\nu}=\nu L \cup\{\nu\} \cup\{\mathbb{I}\}$. Then $L_{\nu}$ is a chain in $E\left(\mathcal{J}_{\lambda}^{\text {cf }}\right)$. Therefore by Proposition 2.7 we get that there exists a maximal chain $L_{\max }$ in $E\left(\mathcal{J}_{\lambda}^{\text {cf }}\right)$ which contains the chain $L_{\nu}$ and $L_{\max }$ is a band of a bicyclic subsemigroup $S$ in $\mathcal{J}_{\lambda}^{\text {cf }}$. Now Corollary 1.32 of [5] implies that $\varepsilon \mathfrak{C} \iota$ for all elements $\varepsilon$ and $\iota$ of the chain $L_{\nu}$. Hence $\nu \mathfrak{C} \mathbb{I}$ and $\alpha \mathfrak{C} \mathbb{I}$ imply that $\nu \mathfrak{C} \alpha$. Therefore all idempotents of the semigroup $\mathcal{J}_{\lambda}^{\text {cf }}$ are $\mathfrak{C}$-equivalent. Since the semigroup $\mathcal{I}_{\lambda}^{\mathrm{ff}}$ is inverse we conclude that quotient semigroup $\mathcal{J}_{\lambda}^{\mathrm{cf}} / \mathfrak{C}$ contains only one idempotent and by Lemma II.1.10 from [13] the semigroup $\mathcal{J}_{\lambda}^{\mathrm{cf}} / \mathfrak{C}$ is a group.

Suppose that $\alpha$ and $\beta$ are distinct non- $\mathcal{H}$-equivalent elements of the semigroup $\mathcal{J}_{\lambda}^{\mathrm{cf}}$ such that $\alpha \mathfrak{C} \beta$. Then Proposition 2.1 implies that at least one of the following conditions holds:

$$
\alpha \alpha^{-1} \neq \beta \beta^{-1} \quad \text { or } \quad \alpha^{-1} \alpha \neq \beta^{-1} \beta .
$$

By Lemma III.1.1 from [13, we have that $\alpha^{-1} \mathfrak{C} \beta^{-1}$. Then $\alpha \alpha^{-1} \mathfrak{C} \alpha \beta^{-1}$ and $\beta \beta^{-1} \mathfrak{C} \alpha \beta^{-1}$ and hence $\alpha \alpha^{-1} \mathfrak{C} \beta \beta^{-1}$. Similarly we get that $\alpha^{-1} \alpha \mathfrak{C} \beta^{-1} \beta$. Then the first part of the proof implies that $\mathfrak{C}$ is a group congruence on $\mathcal{J}_{\lambda}^{\mathrm{cf}}$.

Theorem 2.9. Every non-trivial congruence on the semigroup $\mathcal{J}_{\lambda}^{c f}$ is a group congruence.

Proof. Let $\mathfrak{C}$ be a non-trivial congruence on the semigroup $\mathcal{J}_{\lambda}^{\text {cf }}$. Let $\alpha$ and $\beta$ be distinct $\mathfrak{C}$-equivalent elements of the semigroup $\mathfrak{I}_{\lambda}^{\text {cf }}$. If the elements $\alpha$ and $\beta$ are not $\mathcal{H}$-equivalent then Proposition 2.8 implies the statement of the theorem.

Suppose that $\alpha \mathcal{H} \beta$. Then Theorem 2.20 from [5] implies that without loss of generality we can assume that $\alpha$ and $\beta$ are elements of the group of units $H(\mathbb{I})$ of the semigroup $\mathcal{I}_{\lambda}^{\text {cf }}$ and hence $\mathbb{I C}\left(\beta \alpha^{-1}\right)$. We denote $\gamma=\beta \alpha^{-1}$. Since $\mathbb{I} \neq \gamma$ we conclude that there exists $x_{0} \in \lambda$ such that $\left(x_{0}\right) \gamma \neq x_{0}$. We define $\varepsilon$ to be an identity selfmap of the set $\lambda \backslash\left\{x_{0}\right\}$. Then $\varepsilon \in \mathcal{I}_{\lambda}^{\text {cf }}$ and $(\varepsilon \cdot \mathbb{I}) \mathfrak{C}(\varepsilon \cdot \gamma)$. Since $\left(x_{0}\right) \gamma \neq x_{0}$ we have that Proposition 2.1 (viii) implies that the elements $\varepsilon$ and $\varepsilon \cdot \gamma$ are not $\mathcal{H}$-equivalent. Then by Proposition 2.8 we get that $\mathfrak{C}$ is a group congruence on $\mathcal{J}_{\lambda}^{\mathrm{cf}}$.

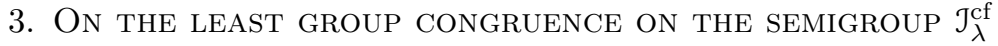

Every inverse semigroup $S$ admits the least group congruence $\sigma$ (see [13, Section III]):

$s \sigma t$ if and only if there exists an idempotent $e \in S$ such that $s e=t e$. 
Theorem 2.9 implies that every non-injective homomorphism $h: \mathcal{J}_{\lambda}^{\mathrm{cf}} \rightarrow S$ from the semigroup $\mathcal{J}_{\lambda}^{\text {cf }}$ into an arbitrary semigroup $S$ generates a group congruence $\mathfrak{h}$ on $\mathcal{J}_{\lambda}^{c f}$. In this section we describe the structure of the quotient semigroup $\mathcal{J}_{\lambda}^{\text {cf }} / \sigma$.

Proposition 3.1. If $\alpha \sigma \beta$ in $\mathcal{J}_{\lambda}^{\mathrm{cf}}$ then

$$
|\lambda \backslash \operatorname{dom} \alpha|-|\lambda \backslash \operatorname{ran} \alpha|=|\lambda \backslash \operatorname{dom} \beta|-|\lambda \backslash \operatorname{ran} \beta| \text {. }
$$

Proof. Let $\varepsilon$ be an idempotent of the semigroup $\mathcal{J}_{\lambda}^{c f}$ such that $\alpha \varepsilon=\beta \varepsilon$. We shall show that the statement of the proposition holds for the elements $\alpha$ and $\alpha \varepsilon$.

Without loss of generality we can assume that $\varepsilon \leqslant \alpha^{-1} \alpha$, i.e., $\operatorname{dom} \varepsilon \subseteq \operatorname{dom}\left(\alpha^{-1} \alpha\right)$. Since $\alpha$ is an injective partial map with $|\lambda \backslash \operatorname{dom} \alpha|<\infty$ and $|\lambda \backslash \operatorname{ran} \alpha|<\infty$, and $\varepsilon$ is an identity map of the cofinite subset $\operatorname{dom} \varepsilon$ in $\lambda$ we conclude that

$$
|\lambda \backslash \operatorname{dom} \alpha|-|\lambda \backslash \operatorname{ran} \alpha|=|\lambda \backslash \operatorname{dom}(\alpha \varepsilon)|-|\lambda \backslash \operatorname{ran}(\alpha \varepsilon)|
$$

This implies the statement of the proposition.

For an arbitrary element $\alpha$ of the semigroup $\mathcal{J}_{\lambda}^{c f}$ we denote

$$
\bar{d}(\alpha)=|\lambda \backslash \operatorname{dom} \alpha| \quad \text { and } \quad \bar{r}(\alpha)=|\lambda \backslash \operatorname{ran} \alpha| .
$$

Proposition 3.2. If $\alpha$ and $\beta$ are arbitrary elements of the semigroup $\mathcal{J}_{\lambda}^{c f}$ then

$$
\bar{d}(\alpha \beta)-\bar{r}(\alpha \beta)=\bar{d}(\alpha)-\bar{r}(\alpha)+\bar{d}(\beta)-\bar{r}(\beta) .
$$

Proof. We consider four cases.

(1) First we consider the case when $\operatorname{ran} \alpha \subseteq \operatorname{dom} \beta$. We put $k=\bar{r}(\alpha)-\bar{d}(\beta)$. Then the definition of the semigroup $\mathcal{J}_{\lambda}^{\mathrm{cf}}$ implies that $k \geqslant 0, \bar{d}(\alpha \beta)=\bar{d}(\alpha), \bar{r}(\alpha \beta)=$ $\bar{r}(\beta)-k$, and hence in this case we get that

$$
\bar{d}(\alpha \beta)-\bar{r}(\alpha \beta)=\bar{d}(\alpha)-\bar{r}(\alpha)+\bar{d}(\beta)-\bar{r}(\beta) .
$$

(2) Suppose that the case when $\operatorname{dom} \beta \subseteq \operatorname{ran} \alpha$ holds. We put $k=\bar{d}(\beta)-\bar{r}(\alpha)$. Then the definition of the semigroup $\mathcal{J}_{\lambda}^{\text {cf }}$ implies that $k \geqslant 0, \bar{d}(\alpha \beta)=\bar{d}(\alpha)+k$, $\bar{r}(\alpha \beta)=\bar{r}(\beta)$, and hence in this case we have that

$$
\bar{d}(\alpha \beta)-\bar{r}(\alpha \beta)=\bar{d}(\alpha)-\bar{r}(\alpha)+\bar{d}(\beta)-\bar{r}(\beta) .
$$

(3) Now we consider the case $(\lambda \backslash \operatorname{ran} \alpha) \cap(\lambda \backslash \operatorname{dom} \beta) \neq \varnothing, \operatorname{ran} \alpha \nsubseteq \operatorname{dom} \beta$ and $\operatorname{dom} \beta \nsubseteq \operatorname{ran} \alpha$. Then the definition of the semigroup $\mathcal{J}_{\lambda}^{\text {cf }}$ implies that there exist positive integers $i, j$ and $k$ such that $i=|(\lambda \backslash \operatorname{ran} \alpha) \backslash(\lambda \backslash \operatorname{dom} \beta)|, j=$ $|(\lambda \backslash \operatorname{ran} \alpha) \cap(\lambda \backslash \operatorname{dom} \beta)|$ and $k=|(\lambda \backslash \operatorname{dom} \beta) \backslash(\lambda \backslash \operatorname{ran} \alpha)|$. Then we have that $\bar{r}(\alpha)=i+j, \bar{d}(\beta)=j+k, \bar{d}(\alpha \beta)=\bar{d}(\alpha)+k$ and $\bar{r}(\alpha \beta)=\bar{r}(\beta)+i$. Therefore, in this case we get that

$$
\bar{d}(\alpha \beta)-\bar{r}(\alpha \beta)=\bar{d}(\alpha)-\bar{r}(\alpha)+\bar{d}(\beta)-\bar{r}(\beta) .
$$

(4) In the case when $(\lambda \backslash \operatorname{ran} \alpha) \cap(\lambda \backslash \operatorname{dom} \beta)=\varnothing$ we have that the definition of the semigroup $\mathcal{J}_{\lambda}^{\mathrm{cf}}$ implies that $\bar{d}(\alpha \beta)=\bar{d}(\alpha)+\bar{d}(\beta), \bar{r}(\alpha \beta)=\bar{r}(\alpha)+\bar{r}(\beta)$, and hence we get that

$$
\bar{d}(\alpha \beta)-\bar{r}(\alpha \beta)=\bar{d}(\alpha)-\bar{r}(\alpha)+\bar{d}(\beta)-\bar{r}(\beta) .
$$

This completes the proof of the proposition.

On the semigroup $\mathcal{J}_{\lambda}^{\text {cf }}$ we define a relation $\sim_{\mathfrak{d}}$ in the following way:

$$
\alpha \sim_{\mathfrak{o}} \beta \quad \text { if and only if } \quad \bar{d}(\alpha)-\bar{r}(\alpha)=\bar{d}(\beta)-\bar{r}(\beta),
$$

for $\alpha, \beta \in \mathcal{J}_{\lambda}^{c f}$. 
Proposition 3.3. Let $\lambda$ be an infinite cardinal. Then $\sim_{\mathfrak{o}}$ is a congruence on the semigroup $\mathcal{I}_{\lambda}^{\mathrm{cf}}$ and moreover the quotient semigroup $\mathcal{J}_{\lambda}^{\mathrm{cf}} / \sim_{\mathfrak{o}}$ is isomorphic to the additive group of integers $\mathbb{Z}(+)$.

Proof. Simple verifications and Proposition 3.2 imply that $\sim_{\mathfrak{d}}$ is a congruence on the semigroup $\mathcal{J}_{\lambda}^{\text {cf }}$. We define a homomorphism $h: \mathcal{J}_{\lambda}^{\text {cf }} \rightarrow \mathbb{Z}(+)$ by the formula $(\alpha) h=$ $\bar{d}(\alpha)-\bar{r}(\alpha)$. Then the definitions of the semigroup $\mathcal{J}_{\lambda}^{\text {cf }}$ and the congruence $\sim_{\mathfrak{d}}$ on $\mathcal{J}_{\lambda}^{\text {cf }}$, and Proposition 3.2 imply that thus defined map $h$ is a surjective homomorphism and moreover $(\alpha) h=(\beta) h$ if and only if $\alpha \sim_{\mathfrak{o}} \beta$ in $\mathcal{J}_{\lambda}^{\text {cf }}$. This completes the proof of the proposition.

Proposition 3.4. Let $\lambda$ be an infinite cardinal. Then for every element $\beta$ of the semigroup $\mathcal{J}_{\lambda}^{\mathrm{cf}}$ such that $\bar{d}(\beta)=\bar{r}(\beta)$ there exists an element $\alpha$ of the group of units of $\mathcal{J}_{\lambda}^{\mathrm{cf}}$ such that $\alpha \sigma \beta$.

Proof. Fix an arbitrary element $\beta$ of the semigroup $\mathcal{J}_{\lambda}^{\text {cf }}$. Without loss of generality we can assume that $\bar{d}(\beta)=\bar{r}(\beta)=k>0$. Let $\left\{x_{1}, \ldots, x_{k}\right\}=\lambda \backslash \operatorname{dom} \beta$ and $\left\{y_{1}, \ldots, y_{k}\right\}=\lambda \backslash \operatorname{ran} \beta$. We define a map $\alpha: \lambda \rightarrow \lambda$ in the following way:

$$
(x) \alpha= \begin{cases}(x) \beta, & \text { if } x \in \operatorname{dom} \beta \\ y_{i}, & \text { if } x=x_{i}, i=1, \ldots, k .\end{cases}
$$

Then $\alpha$ is an element of the group of units of the semigroup $\mathcal{J}_{\lambda}^{\text {cf }}$ and it is obviously that $\alpha \varepsilon=\beta \varepsilon$, where $\varepsilon$ is the identity map of the set $\operatorname{ran} \beta$.

For every $\alpha \in \mathcal{S}_{\lambda}$ we denote $\operatorname{supp}(\alpha)=\{x \in \lambda \mid(x) \alpha \neq x\}$. We define

$$
\mathcal{S}_{\lambda}^{\infty}=\left\{\alpha \in \mathcal{S}_{\lambda} \mid \operatorname{supp}(\alpha) \text { is finite }\right\} .
$$

We observe that the Schreier-Ulam theorem (see [14, Theorem 11.3.4]) implies that $\mathcal{S}_{\lambda}^{\infty}$ is a normal subgroup of $\mathcal{S}_{\lambda}$ and hence $\mathcal{S}_{\lambda} / \mathcal{S}_{\lambda}^{\infty}$ is a group.

Later on, when $\mathfrak{C}$ is a congruence on a semigroup $S$ we shall denote the natural homomorphism generated by the congruence $\mathfrak{C}$ on $S$ by $\pi_{\mathfrak{C}}: S \rightarrow S / \mathfrak{C}$.

The definition of the least group congruence $\sigma$ on the semigroup $J_{\lambda}^{\text {cf }}$ implies the following proposition.

Proposition 3.5. Let $\lambda$ be an infinite cardinal. Then the homomorphic image $(H(\mathbb{I})) \pi_{\sigma}$ of the group of units $H(\mathbb{I})$ of $\mathcal{J}_{\lambda}^{\mathrm{cf}}$ under the natural homomorphism $\pi_{\sigma}: \mathcal{J}_{\lambda}^{\mathrm{cf}} \rightarrow$ $\mathrm{J}_{\lambda}^{\text {cf }} / \sigma$ is isomorphic to the quotient group $\mathcal{S}_{\lambda} / \mathcal{S}_{\lambda}^{\infty}$.

Theorem 3.6. Let $\lambda$ be an infinite cardinal. Then the following conditions hold:

(i) $(H(\mathbb{I})) \pi_{\sigma}=\mathcal{S}_{\lambda} / \mathcal{S}_{\lambda}^{\infty}$ is a normal subgroup of the group $\mathcal{J}_{\lambda}^{\mathrm{cf}} / \sigma$;

(ii) The group $\mathrm{J}_{\lambda}^{\mathrm{cf}} / \sigma$ contains the infinite cyclic subgroup $G$ (i.e., the additive group of integers $\mathbb{Z}(+))$ such that $G \cap \mathcal{S}_{\lambda} / \mathcal{S}_{\lambda}^{\infty}=\{e\}$, where $e$ is the unit of the group $\mathrm{J}_{\lambda}^{\mathrm{cf}} / \sigma$;

(iii) $\mathcal{S}_{\lambda} / \mathcal{S}_{\lambda}^{\infty} \cdot G=\mathcal{J}_{\lambda}^{\mathrm{cf}} / \sigma$.

and hence the group $\mathcal{I}_{\lambda}^{\mathrm{cf}} / \sigma$ is isomorphic to the semidirect product $\mathcal{S}_{\lambda} / \mathcal{S}_{\lambda}^{\infty} \ltimes \mathbb{Z}(+)$.

Proof. (i) Since $\sigma \subseteq \sim_{\mathfrak{d}}$ we conclude that Theorem 1.6 of $[5$ implies that there exists a unique homomorphism $g: J_{\lambda}^{c f} / \sigma \rightarrow G$ such that the following diagram

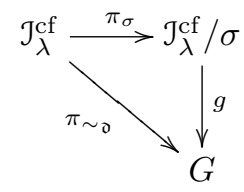


commutes. Then by Proposition 3.5 we have that the homomorphic image $(H(\mathbb{I})) \pi_{\sigma}$ of the group of units $H(\mathbb{I})$ of $\mathcal{J}_{\lambda}^{\text {ct }}$ under the natural homomorphism $\pi_{\sigma}: \mathcal{J}_{\lambda}^{\text {cf }} \rightarrow$ $\mathrm{J}_{\lambda}^{\mathrm{Jff}} / \sigma$ is isomorphic the the quotient group $\mathcal{S}_{\lambda} / \mathcal{S}_{\lambda}^{\infty}$. Now Propositions 3.4 and 3.5 imply that the subgroup $(H(\mathbb{I})) \pi_{\sigma}=\mathcal{S}_{\lambda} / \mathcal{S}_{\lambda}^{\infty}$ of the group $\mathcal{J}_{\lambda}^{\text {cf }} / \sigma$ is the kernel of the homomorphism $g: \mathcal{J}_{\lambda}^{\mathrm{cf}} / \sigma \rightarrow G$, and hence $(H(\mathbb{I})) \pi_{\sigma}=\mathcal{S}_{\lambda} / \mathcal{S}_{\lambda}^{\infty}$ is a normal subgroup of $\mathcal{J}_{\lambda}^{\mathrm{cf}} / \sigma$.

(ii) Fix an arbitrary $\alpha \in \mathcal{J}_{\lambda}^{\text {cf }}$ such that $|\lambda \backslash \operatorname{dom} \alpha|=1$ and $\operatorname{ran} \alpha=\lambda$. Then the definition of the congruence $\sim_{\mathfrak{d}}$ on $\mathcal{J}_{\lambda}^{\text {cf }}$ implies that the element $\alpha^{n}$ is not $\sim_{\mathfrak{d}^{-}}$ equivalent to any element of the group of units $H(\mathbb{I})$ for every non-zero integer $n$, and hence by Proposition 3.5 we get that $\left((\alpha) \pi_{\sigma}\right)^{n} \notin \mathcal{S}_{\lambda} / \mathcal{S}_{\lambda}^{\infty}$. This implies that $\left\{\left((\alpha) \pi_{\sim_{\mathfrak{o}}}\right)^{n} \mid n \in \mathbb{Z}\right\} \cap \mathcal{S}_{\lambda} / \mathcal{S}_{\lambda}^{\infty}=\{e\}$, where $e$ is the unit of the group $\mathcal{J}_{\lambda}^{\text {cf }} / \sigma$. Also, it is obvious that $\left(\alpha^{n}\right) \pi_{\sim_{\mathfrak{o}}}=n \in G$ and $\left\{\left(\alpha^{n}\right) \pi_{\sim_{\mathfrak{o}}} \mid n \in \mathbb{Z}\right\}$ is a cyclic subgroup of $\mathcal{S}_{\lambda} / \mathcal{S}_{\lambda}^{\infty}$.

(iii) Fix an arbitrary element $x$ in $\mathcal{J}_{\lambda}^{\text {cf }} / \sigma$. Let $\xi$ be an arbitrary element of the semigroup $\mathcal{J}_{\lambda}^{\text {cf }}$ be such that $(\xi) \pi_{\sigma}=x$. If $\bar{d}(\xi)=\bar{r}(\xi)$ then by Proposition 3.4 we have that $\xi \sigma \beta$ for some element $\beta$ from the group of units of $\mathcal{J}_{\lambda}^{\text {cf }}$, and hence we get that $x=(\beta) \pi_{\sigma} \cdot e \in \mathcal{S}_{\lambda} / \mathcal{S}_{\lambda}^{\infty} \cdot G$, where $e$ is the unit of the group $\mathcal{J}_{\lambda}^{\text {cf }} / \sigma$. Suppose that $\bar{d}(\xi)-\bar{r}(\xi)=n \neq 0$. Then by Proposition 3.2 we have that $\bar{d}\left(\xi \cdot\left(\alpha^{-1}\right)^{n}\right)-$ $\bar{r}\left(\xi \cdot\left(\alpha^{-1}\right)^{n}\right)=0$. Now, Proposition 3.4 implies that the element $\xi \cdot\left(\alpha^{-1}\right)^{n}$ is $\sigma$-equivalent to some element $\beta$ of the group of units $H(\mathbb{I})$ of $\mathcal{J}_{\lambda}^{\text {cf }}$. Then we have that $\left(\xi \cdot\left(\alpha^{-1}\right)^{n}\right) \pi_{\sigma}=(\beta) \pi_{\sigma}$ and since $\mathcal{J}_{\lambda}^{\text {cf }} / \sigma$ is a group we get that $x=(\xi) \pi_{\sigma}=$ $(\beta) \pi_{\sigma} \cdot\left(\alpha^{n}\right) \pi_{\sigma} \in \mathcal{S}_{\lambda} / \mathcal{S}_{\lambda}^{\infty} \cdot G$. This implies that $\mathcal{S}_{\lambda} / \mathcal{S}_{\lambda}^{\infty} \cdot G=\mathrm{J}_{\lambda}^{\mathrm{cf}} / \sigma$.

The last statement of the theorem follows from statements $(i)-(i i i)$ and Exercise 2.5.3 from [6].

Remark 3.7. Proposition 3.3 implies that for every infinite cardinal $\lambda$ the group $\mathrm{J}_{\lambda}^{\mathrm{cf}} / \sigma$ has infinitely many normal subgroups and hence the semigroup $\mathcal{J}_{\lambda}^{\mathrm{cf}}$ has infinitely many group congruences.

Acknowledgements. This research was supported by the Slovenian Research Agency grants P1-0292, J1-5435, and J1-6721. The authors are grateful to the referee for several useful comments and suggestions.

\section{REFERENCES}

[1] ANDERSEN, O.: Ein Bericht über die Struktur abstrakter Halbgruppen, PhD Thesis, Hamburg, 1952.

[2] ANDERSON, L. W.-HUNTER, R. P.-KOCH, R. J.: Some results on stability in semigroups, Trans. Amer. Math. Soc. 117 (1965), 521-529.

[3] BANAKH, T.-DIMITROVA, S.-GUTIK, O.: The Rees-Suschkiewitsch theorem for simple topological semigroups, Mat. Stud. 31 (2009), 211-218.

[4] BANAKH, T.-DIMITROVA, S.-GUTIK, O.: Embedding the bicyclic semigroup into countably compact topological semigroups, Topology Appl. 157 (2010), 2803-2814.

[5] Clifford, A. H.-PRESTON, G. B.: The Algebraic Theory of Semigroups, Vol. I/II, American Mathematical Society, Providence, Rhode Island, 1961/1967.

[6] DIXON J. D.-MORTIMER B.: Permutation Groups, Springer, Berlin, 1996.

[7] EBERHART, C.-SELDEN, J.: On the closure of the bicyclic semigroup, Trans. Amer. Math. Soc. 144 (1969), 115-126.

[8] GUTIK, O.-REPOVŠ, D.: On countably compact 0-simple topological inverse semigroups, Semigroup Forum 75 (2007), 464-469. 
[9] GUTIK, O.-REPOVŠ, D.: Topological monoids of monotone injective partial selfmaps of $N$ with cofinite domain and image, Stud. Sci. Math. Hung. 48 (2011), 342-353.

[10] GUTIK, O.-REPOVŠ, D.: On monoids of injective partial selfmaps of integers with cofinite domains and images, Georgian Math. J. 19 (2012), 511-532.

[11] HILDEBRANT, J. A.-KOCH, R. J.: Swelling actions of $\Gamma$-compact semigroups, Semigroup Forum 33 (1986), 65-85.

[12] HOWIE, J. M.: Fundamentals of Semigroup Theory, London Math. Soc. Monogr. (N.S.) No. 12, Clarendon Press, Oxford, 1995.

[13] PETRICH, M.: Inverse Semigroups, Pure Appl. Math., John Wiley \& Sons, New York, 1984.

[14] SCOTT, W. R.: Group Theory, Dover Publications, Inc., New York, 1987.

[15] SHELAH, S.-STEPRĀNS, J.: Non-trivial homeomorphisms of $\beta N \backslash N$ without the Continuum Hypothesis, Fund. Math. 132 (1989), 135-141.

[16] SHELAH, S.-STEPRĀNS, J.: Somewhere trivial autohomeomorphisms, J. London Math. Soc. (2), 49 (1994), 569-580.

[17] SHELAH, S.-STEPRĀNS, J.: Martin's axiom is consistent with the existence of nowhere trivial automorphisms, Proc. Amer. Math. Soc. 130 (2002), 2097-2106.

[18] VELIČKOVIĆ, B.: Definable automorphisms of $\mathcal{P}(\omega)$ / fin, Proc. Amer. Math. Soc. 96 (1986), 130-135.

[19] VELIČKOVIĆ, B.: Applications of the Open Coloring Axiom, In Set Theory of the Continuum, H. Judah, W. Just et H. Woodin, eds., Pap. Math. Sci. Res. Inst. Workshop, Berkeley, 1989, MSRI Publications. Springer-Verlag. Vol. 26, Berlin, (1992), pp. 137-154.

[20] VELIČKOVIĆ, B.: OCA and automorphisms of $\mathcal{P}(\omega)$ / fin, Topology Appl. 49 (1993), 1-13.

[21] VAGNER, V. V.: Generalized groups, Dokl. Akad. Nauk SSSR 84 (1952), 1119-1122 (in Russian).

Department of Mechanics and Mathematics

IVAN Franko National University OF LVIV

UNIVERSYTETSKA 1

LVIV, 79000

UKRAINE

E-mail address: o_gutik@franko.lviv.ua

FACUlty OF EduCATion, AND

Faculty of Mathematics and Physics

UNIVERSITY OF LJUBLJANA

KARDELJEVA PLOŠČAD 16

LJUBLJANA, 1000

SLOVENIA

E-mail address: dusan.repovs@guest.arnes.si 